Delft University of Technology

\title{
Numerical Study on Trailing-Edge Noise Attenuation using 3D-Printed Porous Insert
}

Teruna, C.; Avallone, F.; Ragni, D.; Casalino, D.; Rubio Carpio, A.

DOI

10.2514/6.2021-2264

Publication date

2021

Document Version

Final published version

Published in

AIAA AVIATION 2021 FORUM

\section{Citation (APA)}

Teruna, C., Avallone, F., Ragni, D., Casalino, D., \& Rubio Carpio, A. (2021). Numerical Study on TrailingEdge Noise Attenuation using 3D-Printed Porous Insert. In AIAA AVIATION 2021 FORUM [AIAA 20212264] (AIAA Aviation and Aeronautics Forum and Exposition, AIAA AVIATION Forum 2021). https://doi.org/10.2514/6.2021-2264

\section{Important note}

To cite this publication, please use the final published version (if applicable).

Please check the document version above.

\section{Copyright}

Other than for strictly personal use, it is not permitted to download, forward or distribute the text or part of it, without the consent of the author(s) and/or copyright holder(s), unless the work is under an open content license such as Creative Commons.

Takedown policy

Please contact us and provide details if you believe this document breaches copyrights. We will remove access to the work immediately and investigate your claim. 
Green Open Access added to TU Delft Institutional Repository

'You share, we take care!' - Taverne project

https://www.openaccess.nl/en/you-share-we-take-care

Otherwise as indicated in the copyright section: the publisher is the copyright holder of this work and the author uses the Dutch legislation to make this work public. 


\title{
Numerical Study on Trailing-Edge Noise Attenuation Using 3D-Printed Porous Insert
}

\author{
Christopher Teruna *, Francesco Avallone ${ }^{\dagger}$, Daniele Ragni ${ }^{\ddagger}$, Damiano Casalino ${ }^{\S}$, Alejandro Rubio-Carpio ${ }^{\mathbb{I}}$
}

This manuscript presents a numerical investigation of an open-cell 3D-printed permeable/porous insert used to reduce turbulent boundary layer-trailing edge (TBL-TE) noise. The matrix topology of the insert resembles the lattice of diamond atoms, and thus, it is also referred to as the diamond trailing edge (TE). The porous insert replaces the last $20 \%$ of the chord of a NACA 0018 airfoil. The airfoil is set to zero angle of attack and the chord-based Reynolds number equals to $2.8 \times 10^{5}$. The geometrical details of the 3D-printed insert are replicated in the simulation to allow comparison with the corresponding experimental measurements. The diamond $T E$ is found to reduce noise by up to $10 \mathrm{~dB}$ in the low frequency range. At higher frequencies however, the diamond TE causes a slight noise increase. Using a wake survey method, the porous insert is found to cause a minor drag increase compared to its solid counterpart. It is found that the diamond TE produces stronger surface pressure fluctuations, which would have resulted in higher noise intensity according to analytical models. However, by using a source localization method based on the vortex sound theory, it is observed that the increase in pressure fluctuations is primarily due to the exposed pores at the surface of the porous material, which is responsible for the high-frequency excess noise. These analyses also support the argument that a permeable TE produces different acoustic scattering characteristics with respect to the solid TE.

\section{Nomenclature}

$\begin{array}{ll}b & =\text { airfoil span }(\mathrm{mm}) \\ \mathrm{C}_{p} & =\text { mean pressure coefficient } \\ \mathrm{C}_{d} & =\text { mean drag coefficient } \\ c & =\text { chord length }(\mathrm{mm}) \\ h & =\text { sample thickness }(\mathrm{mm}) \\ H & =\text { shape factor } \\ p_{\mathrm{RMS}} & =\text { root-mean-square of pressure fluctuations }(\mathrm{Pa}) \\ q_{\infty} & =\text { freestream dynamic pressure }(\mathrm{Pa}) \\ R_{I} & =\text { inertial resistivity }(1 / \mathrm{m}) \\ R_{V} & =\text { viscous resistivity }(1 / \mathrm{s}) \\ \mathrm{SPL} & =\text { Sound pressure level }(\mathrm{dB} / \mathrm{Hz}) \\ U & =\text { mean velocity in the } x \text { direction }(\mathrm{m} / \mathrm{s}) \\ u_{\mathrm{RMS}} & =\text { root-mean-squre of velocity fluctuations in the } x \text { direction }(\mathrm{m} / \mathrm{s}) \\ V & =\text { mean velocity in the } y \text { direction }(\mathrm{m} / \mathrm{s}) \\ v_{\mathrm{RMS}} & =\text { root-mean-squre of velocity fluctuations in the } y \text { direction }(\mathrm{m} / \mathrm{s}) \\ U_{\infty} & =\text { mean freestream velocity }(\mathrm{m} / \mathrm{s}) \\ \delta^{*} & =\text { boundary layer displacement thickness }(\mathrm{mm}) \\ \delta_{99} & =\text { boundary layer thickness at } 99 \% U_{\infty}(\mathrm{mm}) \\ \theta_{*} & =\text { boundary layer momentum thickness }(\mathrm{mm})\end{array}$

\footnotetext{
* Doctoral candidate, Wind Energy Department, Delft University of Technology, Delft 2629HS, The Netherlands. AIAA Student Member.

$\dagger$ Assistant professor, Wind Energy Department, Delft University of Technology, Delft 2629HS, The Netherlands. AIAA Member.

$¥$ Associate professor, Delft University of Technology, Delft 2629HS, The Netherlands. Wind Energy Department, AIAA Member.

$\S$ Professor, Wind Energy Department, Delft University of Technology, Delft 2629HS, The Netherlands. AIAA Member.

IDoctoral candidate, Wind Energy Department, Delft University of Technology, Delft 2629HS, The Netherlands. AIAA Student Member.
} 


\section{Introduction}

$\mathrm{T}$ URBULENT boundary layer-trailing edge (TBL-TE) noise [1,2] is one of the most relevant noise generation mechanisms for wind turbine blades [3]. The mechanism involves the scattering of unsteady pressure fluctuations underneath a turbulent boundary layer as they convect past the trailing edge [4]. Several techniques to mitigate TBL-TE noise have been reported in the literature, such as the trailing edge serrations [3, 5, 6]. Recently, open-cell porous media have been proposed as alternative approaches for noise reduction [7-10]. Porous medium enables flow transpiration and thus, it can be considered as an intermediary condition between the solid airfoil body and the free fluid. This characteristic is expected to be beneficial for mitigating TE noise as it realizes a milder impedance transition at a trailing edge [11-13].

Applications of porous media to reduce TBL-TE noise have been demonstrated in several studies. Geyer and Sarradj [9] performed experiments on porous airfoils made of different materials. The extent of permeable surface on the airfoil was varied by covering the upstream portion of the porous airfoil with a non-permeable foil. Higher noise reduction could be achieved when larger porous extent was utilized, although this would also deteriorate the aerodynamic performance. The authors concluded that limiting the extent of the permeable surface at the trailing-edge location should offer an acceptable compromise between acoustic and aerodynamic performance. More recently, Rubio-Carpio et al. [10, 14] studied the application of metal-foam trailing-edge insert on a NACA 0018 airfoil. The authors tested inserts made using the same material but with different permeability due to differences in the mean pore diameter. The metal-foam insert was found to reduce noise by up to $10 \mathrm{~dB}$ at low frequency with respect to the solid one, although a slight noise increase $(\approx)$ was observed at high frequency. The noise increase was attributed to the higher surface roughness [7, 15]. In line with the findings of Geyer and Sarradj [9], the metal-foam insert with larger pore size (i.e., higher permeability and porosity) produced larger noise attenuation.

Several investigations have also been dedicated to elucidate the noise reduction mechanism of the porous trailing edge. Delfs et al. [16] suggested that the permeable material enables the flow field on both suction and pressure sides of the airfoil to interact with each other, referred to as a pressure balance process. They observed that there was no noise reduction if one side of the porous trailing edge were covered with a non-permeable tape. A similar study has been performed by Rubio-Carpio et al. [14] when they tested a porous trailing edge insert made of metal-foam. One of the insert was modified by adding a thin layer of non-permeable adhesive along the symmetry plane of the insert, which was subsequently found to completely lose its noise mitigation capability. In a follow-up study [12], the same authors evidenced the pressure release process by demonstrating that turbulent fluctuations in the boundary layer at both sides of the porous insert were correlated, but not for the solid trailing edge.

Despite the aforementioned findings, experimental measurements near and inside the porous medium are relatively difficult to perform [17]. Numerical simulations can be considered as alternatives, for which porous medium models have been developed for [18]. Recently, Teruna et al. [13, 19] carried out numerical simulations that replicate the experimental setup of Rubio-Carpio et al. [14]. The simulation considers an open-cell metal-foam trailing edge that was modeled using an equivalent fluid region governed by the Darcy's law. It was found that the presence of the porous trailing edge had relatively minor effects on boundary layer properties and surface pressure statistics. However, it was implied that the scattering characteristics on the porous trailing edge are different compared to the solid one. The investigation evidenced the presence of a secondary source near the solid-porous junction, which was consistent with the acoustic beamforming maps from the experiment. Moreover, the porous trailing edge realized a milder impedance jump at the trailing edge, leading to a less efficient scattering [4]. It was also suggested that additional acoustic scattering took place along the porous medium surface, which promoted destructive interference among the noise sources. None of the previously mentioned phenomena was observed if the porous insert was made impermeable along the chord line, thus justifying the absence of noise reduction.

While the study of Teruna et al. [13] managed to reveal several aspects of the noise reduction mechanisms of a permeable trailing edge, it is yet to be verified whether previously observed trends were the consequence of complex flow behaviors within the porous medium or were simply artefacts due to the usage of a porous medium model. Hence, it would be desirable to revisit the study with a fully-resolved porous insert geometry. Nevertheless, it is considered to be challenging to numerically replicate the metal-foam insert due to its geometrical elements being much smaller compared to the resolved flow scales. Instead, it would be more feasible to employ an synthetic porous geometry with regular unit cell that allows for direct comparison between the simulation and the experiment. Hence, this manuscript considers a 3D-printed porous trailing edge to study its aeroacoustics behaviors in comparison to those previously observed in simulations utilizing porous-medium modelling approach. In particular, a porous insert whose matrix resembles that of diamond atomic structure has been chosen since it has been found experimentally to produce similar noise attenuation capability as the metal-foam one.

The manuscript is organized as follows. Section III provides a brief description on the porous material characterization 
and the following methodologies, including the simulation setup and an overview of Lattice-Boltzmann method in PowerFLOW. Section IV discusses the simulation results, which is summarized afterward in Section V.

\section{Methodologies}

\section{A. Numerical Solver}

This section provides a brief summary of the numerical technique implemented in the commercial Lattice-Boltzmann solver PowerFLOW 5.4b. For more details on the theoretical foundation of the Lattice-Boltzmann method (LBM), readers are advised to consult Succi [20]. The LBM is based on Boltzmann's kinetic theory of gases which describes the mesoscopic behavior of fluid particles, which is mathematically expressed as follows;

$$
\frac{\partial F}{\partial t}+\boldsymbol{V} \cdot \nabla F=\mathbf{B}
$$

where $F(\boldsymbol{x}, t)$ is the particle distribution function in spatial $(\boldsymbol{x})$ and temporal space $(t), \boldsymbol{V}$ is the particle velocity, and $\mathbf{B}$ is the collision operator. The equation is then discretized on a cartesian grid, referred to as a lattice, where the fluid motion is limited to a set of discrete dimensions and vectors. PowerFLOW employs the D3Q19 model for solving low Mach number cases [21-23]. The discretized form of the LBM is written as follows;

$$
F_{\mathrm{n}}\left(\boldsymbol{x}+\boldsymbol{V}_{\mathrm{n}} \Delta t, t+\Delta t\right)-F_{\mathrm{n}}(\boldsymbol{x}, t)=\mathbf{B}_{\mathrm{n}}(\boldsymbol{x}, t)
$$

where $F_{\mathrm{n}}$ is the particle distribution function in $\mathrm{n}^{\text {th }}$ direction in the lattice, $\boldsymbol{V}_{\mathrm{n}}$ is the discrete particle velocity in $\mathrm{n}^{\text {th }}$ direction. The collision term $\mathbf{B}_{\mathrm{n}}$ follows the Bhatnagar-Gross-Krook model [24];

$$
\mathbf{B}_{\mathrm{n}}=-\frac{\Delta t}{\tau}\left[F_{\mathrm{n}}(\boldsymbol{x}, t)-F_{\mathrm{n}}^{\mathrm{eq}}(\boldsymbol{x}, t)\right]
$$

where $\tau$ is the relaxation time which is a function of fluid viscosity and temperature, while $F_{\mathrm{n}}^{\text {eq }}$ is the equilibrium distribution function can be approximated with a second-order expansion as follows [23];

$$
F_{\mathrm{n}}^{\mathrm{eq}}=\rho \omega_{\mathrm{n}}\left[1+\frac{\boldsymbol{V}_{\mathrm{n}} \boldsymbol{u}}{a_{s}^{2}}+\frac{\left(\boldsymbol{V}_{\mathrm{n}} \boldsymbol{u}\right)^{2}}{2 a_{s}^{4}}-\frac{|\boldsymbol{u}|^{2}}{2 a_{s}^{2}}\right]
$$

where $\omega_{\mathrm{n}}$ are the weighting functions based on the D3Q19 model, and $a_{s}=\frac{1}{\sqrt{3}}$ is the non-dimensional speed of sound within the lattice.

Solving Eq. 2 for obtaining $F(\boldsymbol{x}, t)$ allows the recovery of macroscopic flow quantities, such as density $\rho$ and velocity $\vec{u}$ which are mathematically expressed as follow;

$$
\begin{aligned}
\rho(\boldsymbol{x}, t) & =\sum_{\mathrm{n}} F_{\mathrm{n}}(\boldsymbol{x}, t) \\
\rho \boldsymbol{u}(\boldsymbol{x}, t) & =\sum_{\mathrm{n}} \boldsymbol{V}_{\mathrm{n}} F_{\mathrm{n}}(\boldsymbol{x}, t)
\end{aligned}
$$

The simulation domain has been discretized into cubic volumetric elements, also referred to voxel (i.e., volumetric pixel). The dimension of each voxel may be adjusted accordingly where detailed flow description is necessary (e.g., in the vicinity of a wall with arbitrary geometry). The domain is then subdivided into smaller regions in which voxel dimensions of adjacent region is allowed to vary by a factor of 2. A Very Large Eddy Simulation (VLES) model based on two-equations $k-\epsilon$ Renormalization Group (RNG) [25] has been adopted. A wall function is applied to the wall-adjacent grid, which is based on generalized law-of-the-wall model [26], extended by considering the effect of pressure gradient and surface roughness. For computing far-field noise, PowerFLOW employs Ffowcs-Williams \& Hawking (FW-H) analogy [27] based on Farrasat's formulation 1A [28] with forward-time solution [29], extended for application on permeable integration surfaces. 


\section{B. Macroscopic Transport Phenomena in Porous Materials}

Although there are as many as 6 different parameters that can be used to characterize the macroscopic properties of transport phenomena in porous materials [30], those that are considered relevant for aeroacoustics purposes include porosity, permeability, and form coefficient [10, 15, 31]. The porosity of the material is defined as:

$$
\phi=1-\frac{\rho_{p}}{\rho_{s}}
$$

where $\rho_{p}$ and $\rho_{s}$ are density of the porous material sample and that of the solid (non-permeable) sample, respectively. In essence, the porosity represents the volume of a porous material that constitutes of void (empty space) that can be occupied by fluid. Permeability $K$ and form coefficient $C$ are defined using the Hazen-Dupuit-Darcy equation [30], which collectively defines the amount of resistance encountered by the flow inside the porous medium.

$$
\frac{\Delta p}{h}=\frac{\mu_{\infty}}{K} v_{d}+\rho_{\infty} C v_{d}^{2}
$$

where the left hand side describes a pressure gradient $\Delta p$ along a a porous material sample of thickness $h$. At the right hand side, $\mu_{\infty}$ and $\rho_{\infty}$ are the fluid's freestream dynamic viscosity and density respectively.

The Hazen-Dupuit-Darcy equation forms a second order polynomial in term of Darcian velocity $v_{d}$ that is the spatially-averaged flow velocity in the porous medium. The linear term of the equation describes the momentum loss due to viscous effect, and thus the coefficient $\mu_{\infty} / K$ is also referred to as viscous resistivity $R_{V}$. Meanwhile the second term describes the inertial resistance related to local flow acceleration within the pores, and thus the form coefficient $C$ is also referred to as inertial resistivity $R_{I}$. As implied, the contribution of inertial resistivity becomes significant when high Reynolds number flow permeates the porous medium [30].

The present study considers a porous material that consists of cubic unit cells. The unit cell has a dimension is $6.36 \mathrm{~mm}$, in which cylindrical struts form a matrix in a lattice that resembles diamond atom arrangement. As such, it is referred to as the diamond (DMND for short) material for the rest of this manuscript. The geometrical properties of the unit cell of the diamond material are reported in Table 1 .

Table 1 The geometrical properties of the diamond material unit cell.

\begin{tabular}{ccccc} 
& Unit cell size $(\mathrm{mm})$ & Pore size $(\mathrm{mm})$ & Matrix strut thickness $(\mathrm{mm})$ & Matrix strut length $(\mathrm{mm})$ \\
\hline Diamond & 6.36 & 1.20 & 0.52 & 2.60
\end{tabular}

\section{Flow Characterization of a 3D-Printed Porous Material}

For obtaining the viscous and inertial resistivity of the porous material, an experimental characterization rig has been prepared [10]. The rig consists of a straight tube of $1.5 \mathrm{~m}$ long, in which a test section containing the porous material sample is added at the center. The inner diameter of the tube is $50 \mathrm{~mm}$ and the tube inlet is connected to a reservoir of pressurized air while the outlet is exposed to ambient atmosphere. There are two pressure taps are located $50 \mathrm{~mm}$ upstream and downstream of the test section, connected to a Mensor 2101 differential pressure sensor (range up to $15 \mathrm{kPa}$, accuracy $- \pm 1 \mathrm{~Pa}$ ). Mass flow rate in the tube is controlled using an Aventics pressure regulator and a TSI 4040 volumetric flow-meter located upstream the tube inlet. This setup has been previously used for characterizing other types of porous material samples [10, 32].

The experimental characterization rig is also replicated numerically as shown in Fig. 1. The tube inlet is specified with a freestream velocity that equals the Darcian velocity $\left(v_{d}\right)$, while the tube outlet is prescribed with the atmospheric pressure $p_{\infty}$. The inner wall of the tube has a no-slip boundary condition. The diamond material sample is placed at the center of the tube. Note that the geometrical features of the diamond material is fully-resolved in the simulation. Moreover, it has been verified that assigning 10 voxels across a pore diameter is sufficient to obtain a converged pressure drop across the porous medium. The simulation is carried out for a flow passage between the tube inlet and the tube outlet excluding an initial transient. When considering $v_{d}=2.5 \mathrm{~m} / \mathrm{s}$ and a $10 \mathrm{~mm}$-thick sample, the simulation requires $80.6 \times 10^{3} \mathrm{CPU}$ hours when carried out on a 480-core Intel Xeon Gold 6130 platform. 


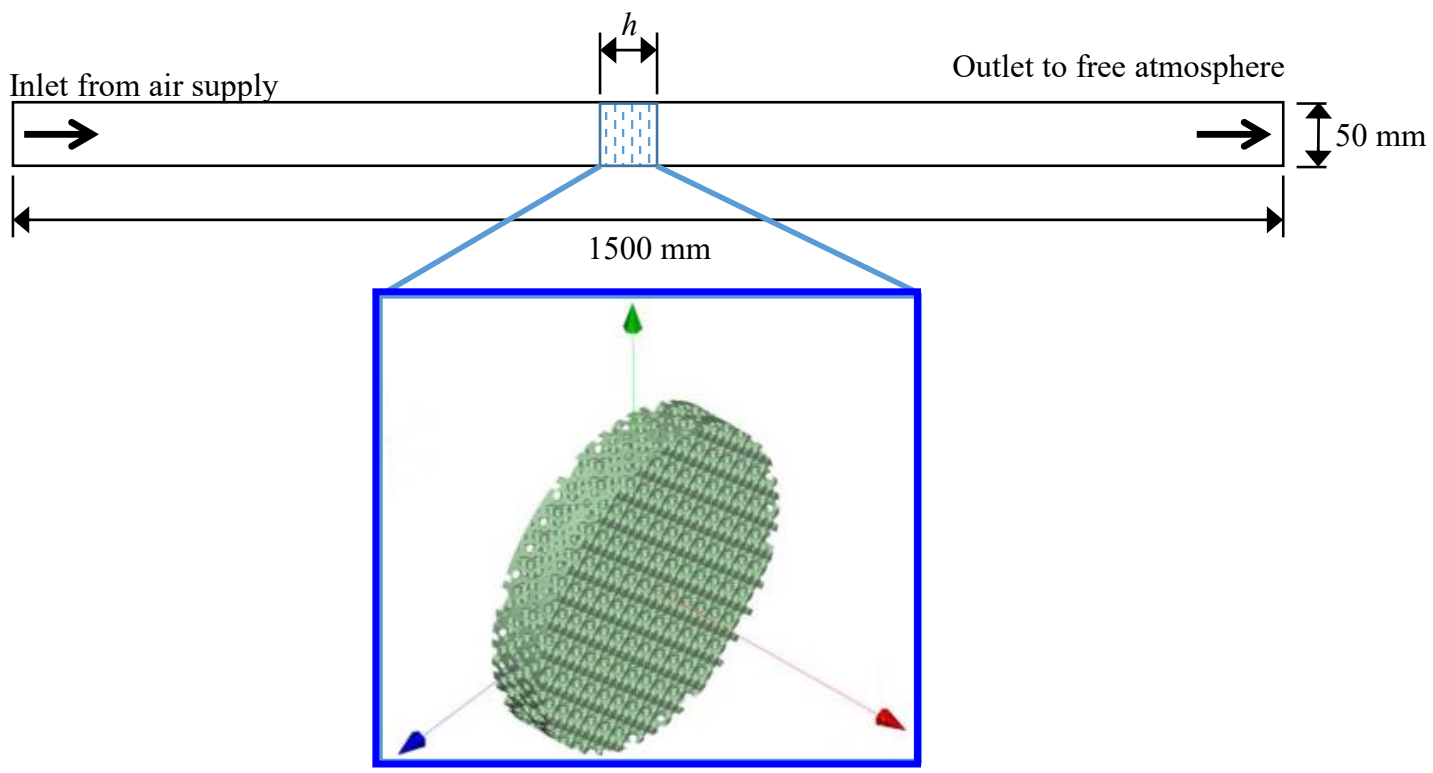

Fig. 1 A sketch of the porous material characterization test rig (not to scale). The inset shows the CAD drawing of the diamond material sample.

\section{Simulation Setup for Trailing-Edge Noise Case}

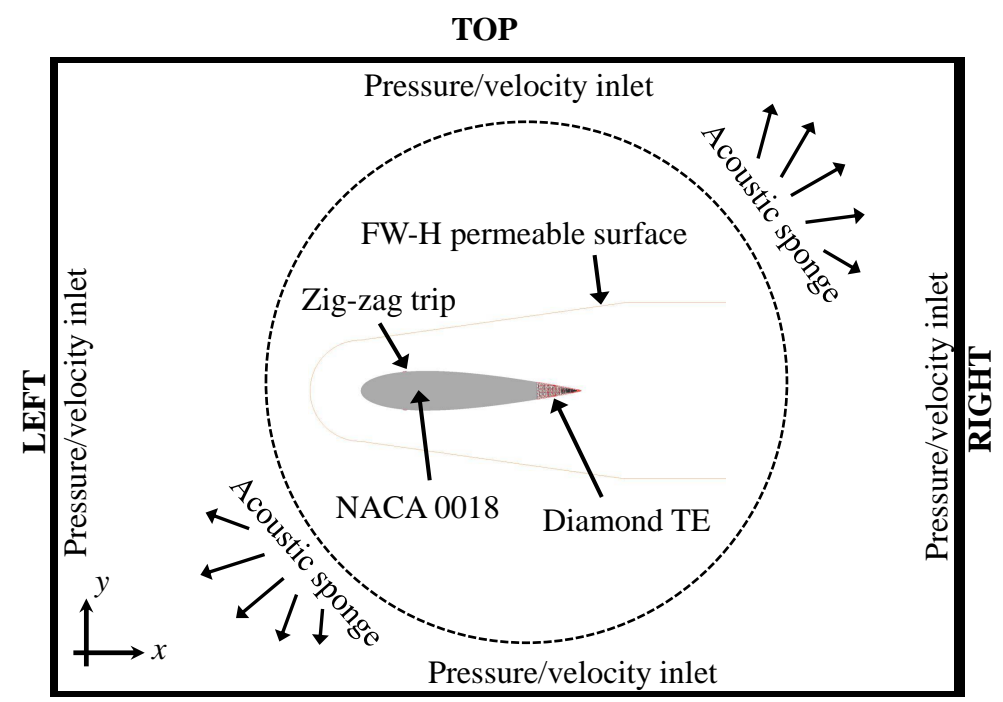

BOTTOM

Fig. 2 A sketch of the computational domain for diamond porous trailing edge noise measurement simulation (boundaries are not to scale).

The simulation setup is similar the one previously used by Teruna et al. [19]. It considers a NACA 0018 airfoil with the chord length $(c)$ of $200 \mathrm{~mm}$ and span $(b)$ of $76.37 \mathrm{~mm}$; the span has been specified to ensure the spanwise periodicity of the unit cell of the porous trailing edge. The airfoil is placed in a rectangular domain that is $100 c$ long and tall, and its width is equal to $b$. The freestream velocity is $U_{\infty}=20 \mathrm{~m} / \mathrm{s}$ (i.e., freestream Mach number $M_{\infty}=0.06$ ), corresponding to a chord-based Reynolds number $\mathrm{Re}_{c}$ of $2.8 \times 10^{5}$. The freestream turbulence intensity is set to $0.1 \%$, and the laminar-turbulent transition is forced using zig-zag strips with the trip thickness of $h_{\text {trip }}=0.6 \mathrm{~mm}$, the trip amplitude of $l_{\text {trip }}=0.3 \mathrm{~mm}$, and the wavelength of $\lambda_{\text {trip }}=3 \mathrm{~mm}$ [21]. The zig-zag strip is installed on both sides of the 
airfoil at $20 \%$ of the chord length. When applicable, the diamond trailing edge (DMND TE) replaces the last $20 \%$ of the airfoil chord.

(a) Near zig-zag trip
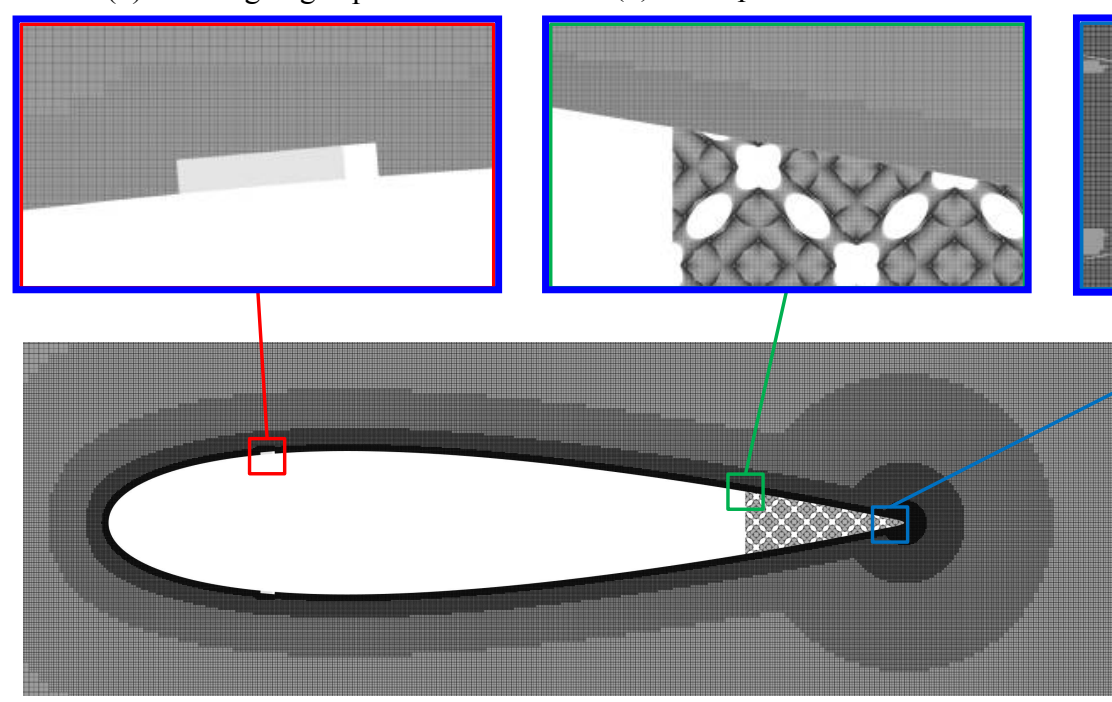

(c) Trailing edge

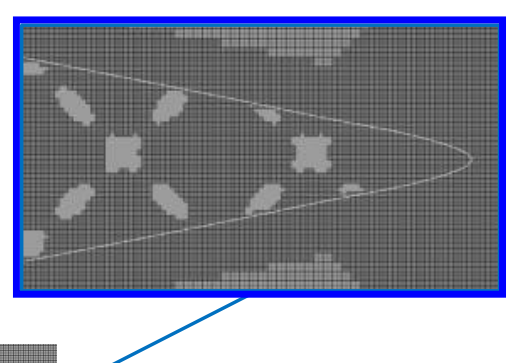

(d) Diamond unit cell

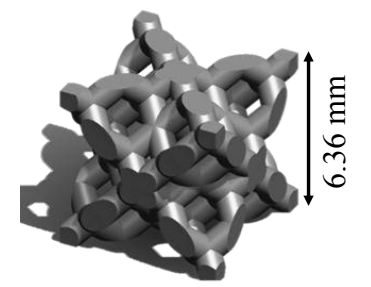

Fig. 3 A view of the computation grid arrangement on the $x-y$ plane. Insets are also provided portraying various regions of interest.

A sketch of the computational domain is shown in Fig. 2. The global coordinate system is also defined in the figure, in which the $x$ and $y$ axes are aligned with the freestream direction and its normal respectively. The origin is located at the midspan of the trailing edge (i.e., $x / c=0$ coincides with the trailing edge), and thus the airfoil leading edge is located at $x / c=-1$ and the airfoil spans in between $-0.191<z / c<0.191$. Freestream pressure and velocity is prescribed at the left, top, right, and bottom domain boundaries, as shown in Fig. 2 Periodic boundary conditions are imposed on the lateral faces of the domain. All solid surfaces on the airfoil, including the struts in the diamond TE and the tripping elements, are no-slip walls. An acoustic sponge region is applied to dampen waves propagating from the source region, starting from a radius of $36 c$ from the trailing edge. The domain is subdivided into 10 grid refinement regions with the finest one located adjacent to the airfoil surface. For the finest grid configuration, the first wall-adjacent cell corresponds to the $y^{+}=3$ at the trailing edge of the solid airfoil. The grid distribution resulting from the discretization procedure is shown in Fig. 3 The simulation is carried out for 20 flow passes with respect to the airfoil chord, for which, it requires $311 \times 10^{3}$ CPU hours when carried out on a 480-core Intel Xeon Gold 6130 platform.

\section{Results and Discussions}

\section{A. Diamond Material Characterization}

The resistivity of the porous material is obtained by curve-fitting Eq. 8 to a series of pressure drop data. The pressure difference across the porous material $(d P / h)$ is measured for different mass flow rate in the tube $\left(v_{d}\right)$. Moreover, several sample thicknesses are tested to assess the trend of the resistivity values. It has been reported in the literature [10, 33] that the resistivity of a porous material converges to a certain value for a sufficiently thick sample. This is due to the increasing influence of the entrance/exit effect (i.e., flow unsteadiness within the first pore distance away from the surface) as the sample thickness approaches that of the unit-cell dimension [34].

The pressure drop trend for the different DMND samples are shown in Fig. 4 (a). Note that the sample thickness is expressed in multiples of unit-cell dimension to ensure geometrical periodicity of the porous material samples. For all sample thicknesses, the pressure drop distributions follow a parabolic trend, although the curves tend to collapse at larger thickness values. This also implies the converging trend of $R_{V}$ and $R_{I}$ as depicted in Fig. $4(b)$. The plot also shows that $R_{V}$ is relatively unaffected with the increasing thickness while $R_{I}$ converges for $h>55 \mathrm{~mm}$. However, the 
(a)

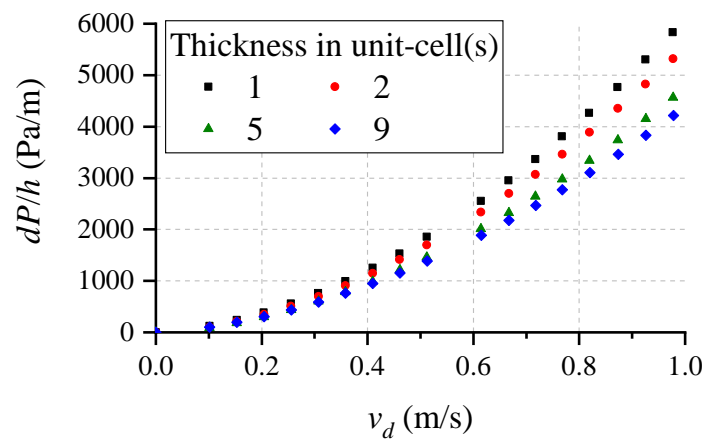

(b)

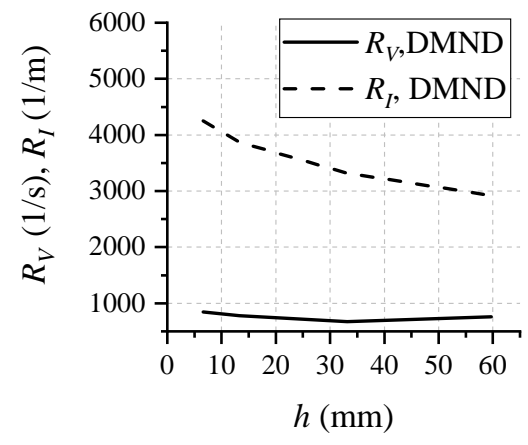

Fig. 4 Plots of $(a)$ pressure drop trend versus different Darcian velocity and $(b)$ the variation of viscous $\left(R_{V}\right)$ and inertial $\left(R_{I}\right)$ resistivity with sample thickness.

converged resistivity value might be irrelevant considering that the maximum thickness of the DMND TE insert is $16 \mathrm{~mm}(\approx 2.5$ unit-cells $)$.

The simulation for characterizing the DMND material has been performed for sample thickness of $10 \mathrm{~mm}$ and $v_{d}=2.75 \mathrm{~m} / \mathrm{s}$. Such a thin sample has been considered as it is expected to be dominated by the entrance/exit effect and therefore the flow field near the porous medium surface has to be well-resolved to capture such effect accurately. The higher Darcian velocity is considered to accelerate numerical convergence and to reduce computational cost, but it also has the consequence of emphasizing the role of the inertial term in Eq. 8 rather than the viscous one. Since $R_{I}$ exhibits more noticeable variation with respect to sample thickness, this particular case serves as a good sensitivity check on the numerical solution.

The viscous and inertial resistivities for this particular sample thickness are presented in Table 2 Considering that the thickness is not an exact multiple of the unit-cell, both $R_{V}$ and $R_{I}$ are extrapolated from Fig. $4(b)$. Regardless, it is found that the pressure drop obtained from the simulation is in good agreement with the prediction using the Hazen-Dupuit-Darcy equation (i.e., Eq. 8). The result also evidences that the 3D-printed DMND geometry and the original CAD model that is employed in the simulation are still comparable in term of quality.

Table 2 Comparison of pressure drop between simulation and the prediction from Eq. 8

\begin{tabular}{|c|c|c|c|c|c|c|c|}
\hline$h(\mathrm{~mm})$ & $\phi$ & $R_{V}(1 / \mathrm{s})$ & $R_{I}(1 / \mathrm{m})$ & $v_{d}(\mathrm{~m} / \mathrm{s})$ & $d P / h(\mathrm{LBM}, \mathrm{Pa} / \mathrm{m})$ & $d P / h($ Eq. & Difference (\%) \\
\hline 10 & 0.5 & 813.2 & 4063 & 2.75 & 39720 & 398 & 3.3 \\
\hline
\end{tabular}

\section{B. Trailing-Edge Noise Measurement}

Far-field noise has been predicted using the solid-surface $\mathrm{FW}-\mathrm{H}$ formulation, which rely on the pressure fluctuations sampled on the surface of the airfoil and the porous material matrix. Data are sampled at a rate of $32 \mathrm{kHz}$ for 20 flow passes. The power spectral density (PSD) of the sound pressure is estimated using the Welch's periodogram method [35] with Hanning window to obtain spectra with a frequency resolution of $100 \mathrm{~Hz}$. The acoustics response of the DMND TE at a location directly above the trailing edge location $(x / c=0, y / c=7.4)$ is plotted in Fig. 5, where the sound pressure level (SPL) has been normalized with a reference pressure of $20 \times 10^{-5} \mathrm{~Pa}$ and a reference distance of $1 \mathrm{~mm}$. The Strouhal number based on the airfoil chord $\left(S t_{c}\right)$ is used as non-dimensional frequency. In plot $(a)$, the simulation result is compared against the experimental one. Since the airfoil span in the simulation is smaller than that in the experiment, the SPL is scaled accordingly to allow comparison, as in Eq. 9 .

$$
\mathrm{SPL}_{\text {scaled }}=\mathrm{SPL}+10 \log _{10}\left(b_{\text {exp }} / b_{\text {sim }}\right)
$$

where $b_{\text {exp }}$ and $b_{\text {sim }}$ are the airfoil span in the experiment and simulation respectively. Plot $(a)$ shows that the spectral features of the DMND TE measured in the experiment have been well captured by the present simulation. 
(a)

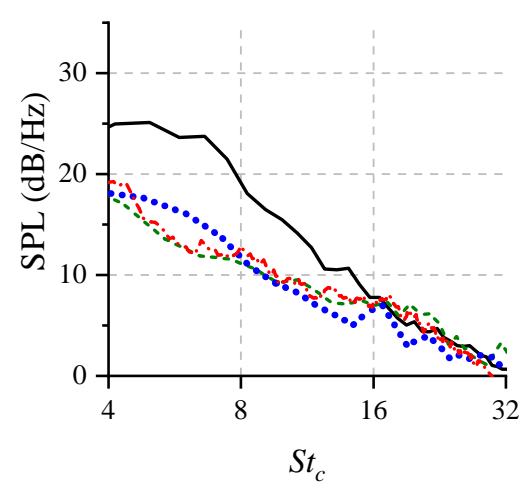

(b)

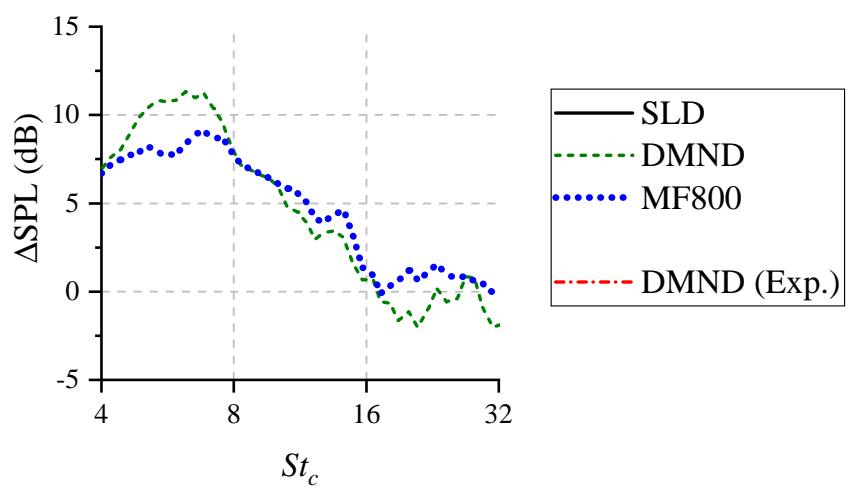

Fig. 5 The far-field noise spectra measured at $x / c=0$ and $y / c=7.4$. Comparison between the simulation and experimental results for the diamond (DMND) case is shown in plot $(a)$ while the noise reduction spectra relative to the solid (SLD) TE case are given in (b) as $\triangle \mathrm{SPL}=\mathrm{SPL} \mathrm{SLD}_{-}-\mathrm{SPL}_{\mathrm{DMND} \mid \mathrm{MF} 800}$. Note that a porous medium model is used for simulating the metal foam (MF800) case.

(a) Solid TE

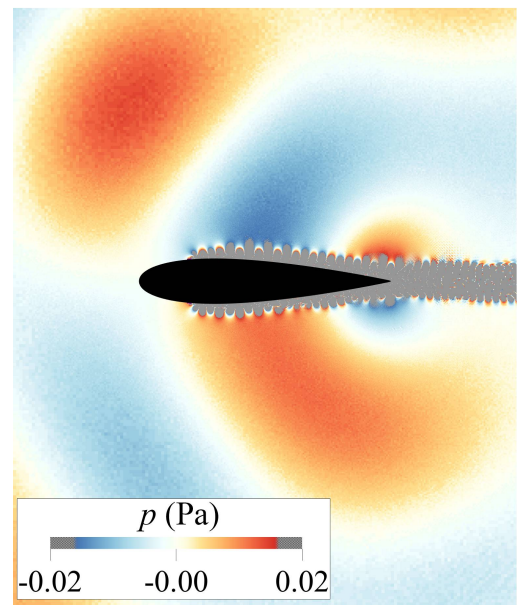

(b) Diamond TE

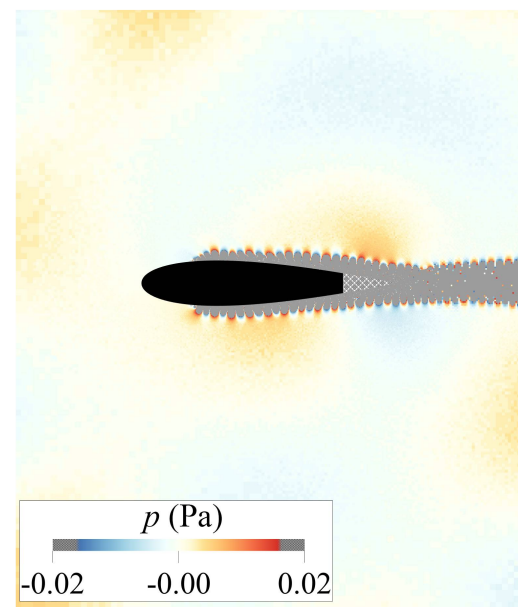

Fig. 6 Acoustic pressure visualization at a $1 / 3$ octave band centered at $1.25 \mathrm{kHz}\left(S t_{c}=12.5\right)$. Pressure fluctuations in the boundary layer and the airfoil wake are masked in grey.

Based on the noise reduction spectra in Fig. 5 $(b)$, the plot can be subdividied into three distinct frequency regions. The first is for $S t_{c}<8$, where the DMND TE reduces noise by an average of $10 \mathrm{~dB}$, the largest within thge present range of frequencies. Then, there is a transition region in between $8<S t_{c}<16$ where the noise reduction becomes smaller up to a cross-over frequency at $S t_{c}=16$. Above this frequency, the DMND TE exhibits a slight noise increase. These behaviors are similar with those observed for the metal-foam (MF800) porous trailing edge previously studied in ref. [11], suggesting that the high frequency noise increase is linked to the geometrical features of the porous trailing edge. This is further corroborated by a previous simulation [19], where it was found that the excess high frequency noise was underpredicted if the porous material were modelled using an equivalent fluid region with smooth interface.

The acoustic field visualization is provided in Fig. 6. The figure shows the contour of pressure fluctuations $p$ bandpass-filtered at a one-third octave band centered at $S t_{c}=12.5$ (i.e., between 1100 and $1400 \mathrm{~Hz}$ ). For solid TE, the sound waves radiate following a cardioid pattern that can be traced back to the trailing edge of the airfoil. Differently, the sound waves produced by the DMND TE appears to have originated from a more upstream position. The contour of DMND TE clearly shows that the far-field noise intensity is lower compared to that of the solid TE, since the frequency band is still located in the transition region of the sound spectra. 
(a) LBM vs Experiment (SLD)

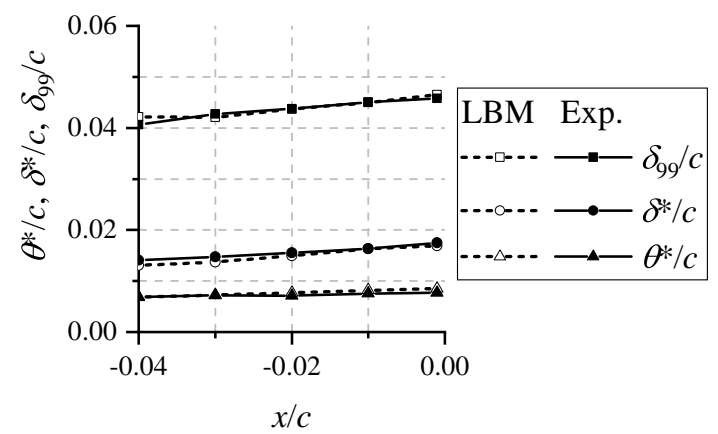

(b) SLD vs DMND

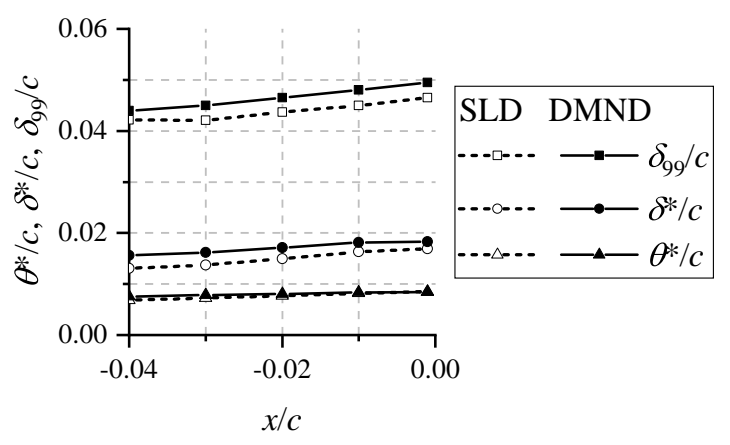

Fig. 7 The trends of integral boundary layer parameters for the last $4 \%$ of the airfoil. Comparison between the simulation and experimental results for the SLD case is shown in plot $(a)$ while the comparison between the SLD and DMND cases are shown in $(b)$.

Table 3 Comparisons of boundary layer parameters at the trailing edge $(x / c=0)$.

\begin{tabular}{r|cccc}
\hline \hline & $\delta_{99}(\mathrm{~mm})$ & $\delta^{*}(\mathrm{~mm})$ & $\theta^{*}(\mathrm{~mm})$ & $H$ \\
\hline Solid trailing edge (SLD) & & & & \\
LBM-VLES, (Teruna et al. [19]) & 9.33 & 3.37 & 1.61 & 2.09 \\
Experiment (Rubio-Carpio et al. [14]) & 9.30 & 3.52 & 1.59 & 2.21 \\
Experiment (Arce-Leon et al. [36]) & 9.40 & 2.10 & 1.30 & 1.62 \\
\hline Diamond trailing edge (DMND) & & & & \\
LBM-VLES, (Present) & 9.90 & 3.65 & 1.69 & 2.16 \\
\hline \hline
\end{tabular}

\section{Flow Field Statistics and Aerodynamic Forces}

The flow field surrounding the trailing edge region of the airfoil is investigated to observe the effects of DMND TE on aerodynamics. Table 3 shows a comparison of the boundary layer parameters between the SLD and DMND TE. Results for the SLD case has been taken from a previous numerical study of the author [19]. The table shows that the simulation result is comparable to other experimental studies, which is also evident in Fig. 7) Compared to the SLD case, the DMND treatment produces a thicker boundary layer, which is in line with the trend when other types of porous material were used [9, 14]. Nevertheless, the boundary layer growth rate of the DMND TE appears to be similar with the SLD one.

Comparisons of velocity profiles for the SLD and DMND cases are plotted in Fig. 8 , The profiles are sampled at two locations along the airfoil midspan: $(a)$ at the trailing edge and $(b)$ in the airfoil wake. Compared to that of the SLD, the DMND one shows a more pronounced velocity deficit at the lower part of the boundary layer. Note that the $U$ profile of the DMND does not reach zero at the wall because the location happens to be on an open pore. The DMND shows enhanced velocity fluctuations compared to the baseline SLD throughout the boundary layer. This process can be attributed to the enhanced turbulent generation process near the porous medium surface due to increased wall shear [37] and surface roughness [38]. The $v_{\mathrm{RMS}}$ of the DMND TE is still relatively high near the surface of porous medium, whereas this value tends toward zero for the non-permeable surface of the SLD TE. The trends of the velocity profiles remain the same in the airfoil wake as depicted in plot $(b)$, in which the mean velocity deficit caused by the DMND TE is still noticeable, while the velocity fluctuations level is higher than that of the SLD TE.

The flow field contours at the midspan of the SLD and DMND TE are illustrated in Fig. 9. Plots $(a)$ and $(b)$ show the mean streamwise and wall-normal velocity components. It is evident that the flow field inside the porous medium is dominated by re-circulation regions since $U$ tends towards negative values. Nonetheless, the mean velocity in the pores is significantly lower than the freestream velocity (i.e., $\approx O\left(10^{-2}\right)$ of $U_{\infty}$ ). The regions closer to the trailing edge tend to show higher velocity since the flow resistance is lower where the porous material is thinner. In the same figure, plots $(c)$ and $(d)$ show the root-mean-square of the velocity fluctuations. Flow fluctuations in the porous medium are of similar 
(a) $x / c=0$
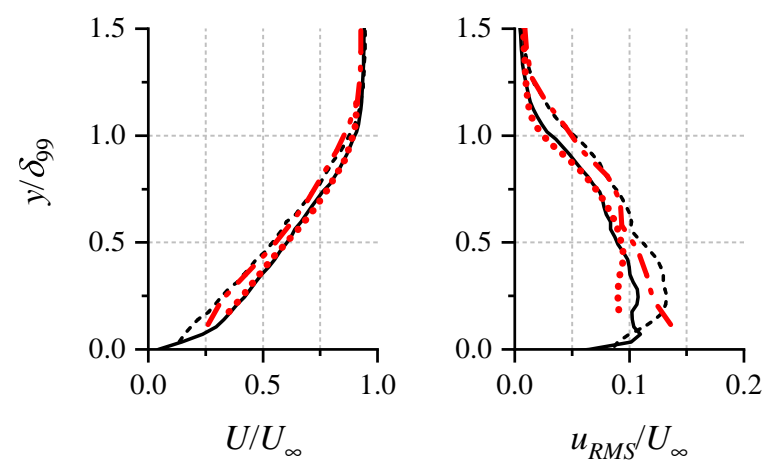

(b) $x / c=0.02$
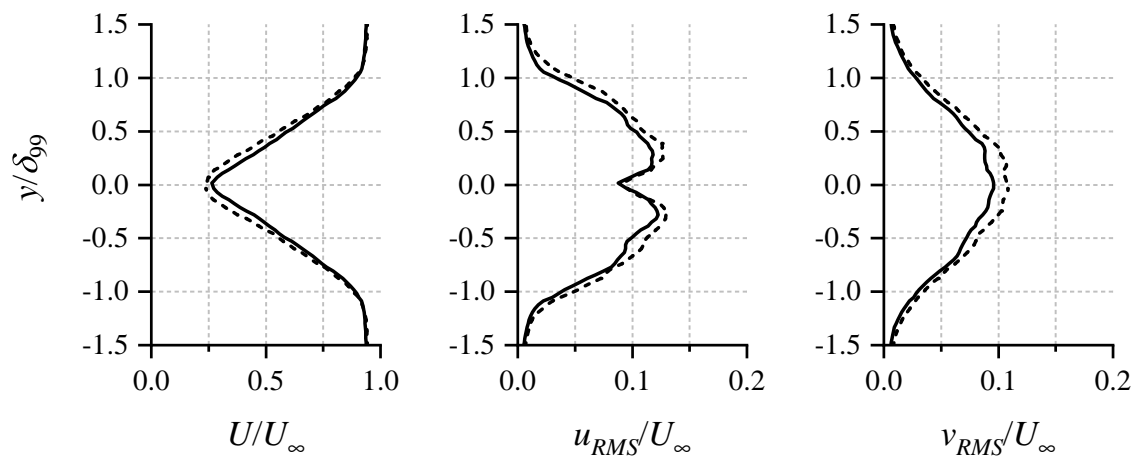

...... SLD (Experiment)

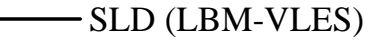

$-\cdot-$ DMND (Experiment)

Fig. 8 The boundary layer profiles at $(a)$ the trailing edge, and $(b)$ the wake region sampled at the airfoil midspan. The velocity statistics are normalized with $U_{\infty}$, while the $y$ coordinate with the $\delta_{99}$ of the SLD case. 
(a)

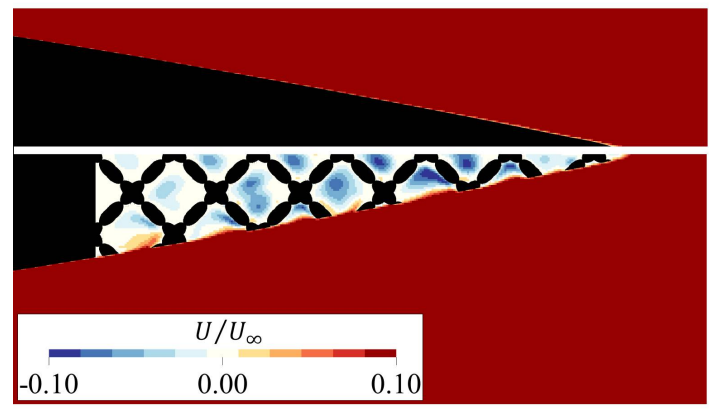

(c)

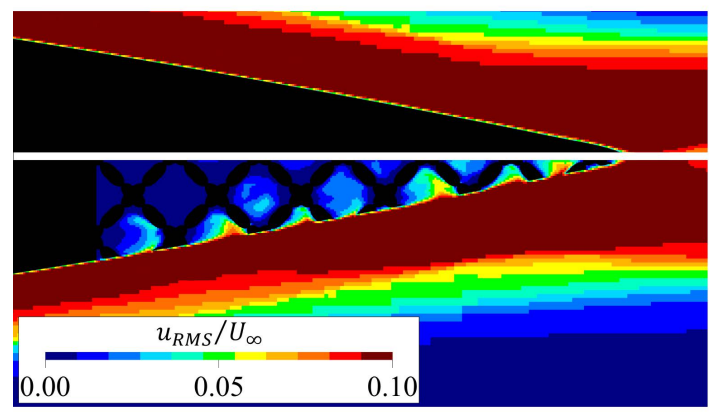

(e)

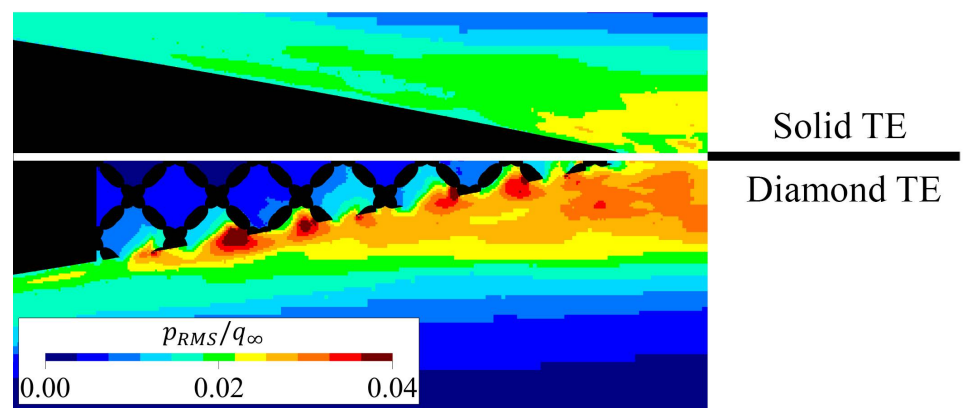

(b)

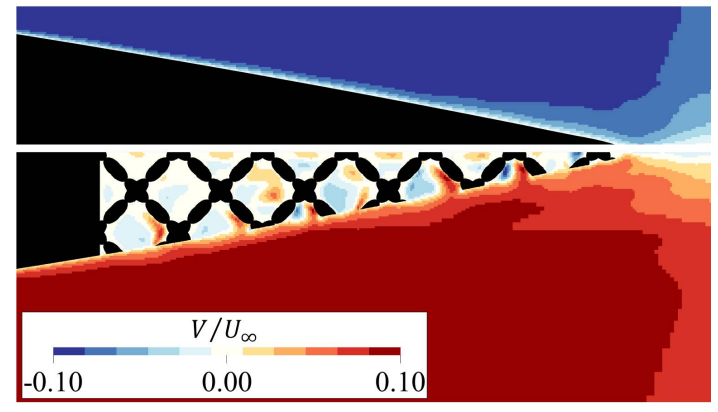

$(d)$

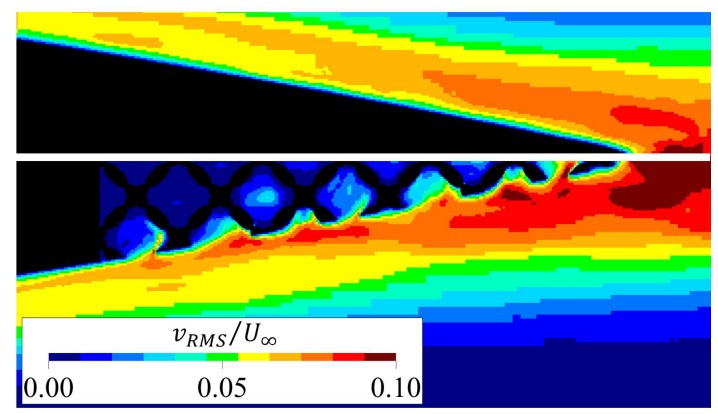

Fig. 9 Flow field contours sampled at the airfoil midspan. Plots $(a)$ and $(b)$ depict the mean streamwise and normal velocity components; the root-mean-square of the velocity fluctuations are shown in plots $(c)$ and $(d)$. Plot (e) shows the root-mean-square of the pressure fluctuations. 
Table 4 The comparison of drag coefficient estimate between the solid and diamond TE cases.

\begin{tabular}{lcc} 
& Solid TE & Diamond TE \\
\hline$C_{d \text {,mean }}$ & 0.0240 & 0.0262 \\
$\Delta C_{d}$ from solid TE & - & $+8.9 \%$
\end{tabular}

(a)

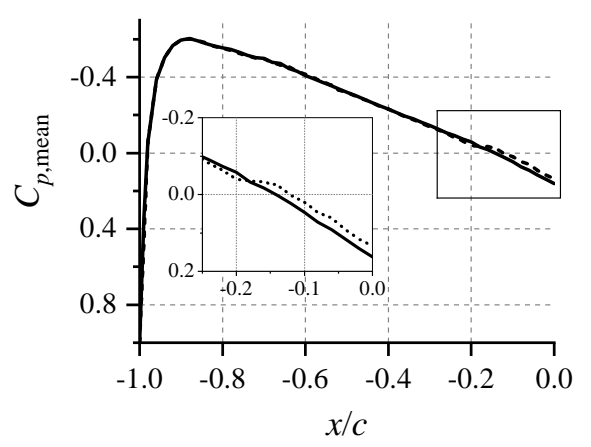

(b)

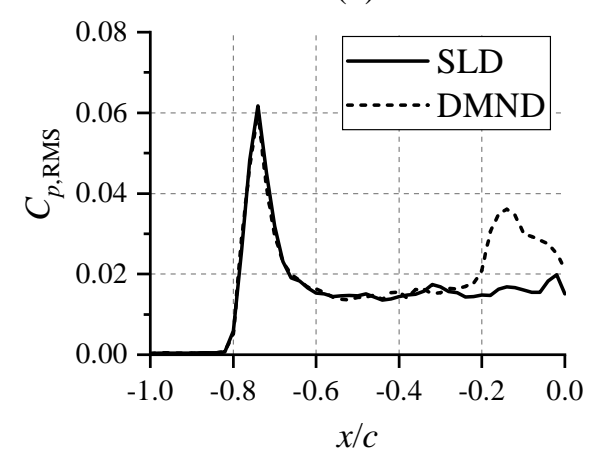

Fig. 10 Spanwise-averaged mean surface pressure distribution (a) and the root-mean-square (RMS) of surface pressure fluctuations (b) on the NACA 0018 with solid and diamond trailing edge. $C_{p, \mathrm{RMS}}$ is normalized with freestream dynamic pressure, i.e., $0.5 \rho_{\infty} U_{\infty}^{2}$. Discontinuities in the plots near the tripping location and open pores at the TE have been replaced with interpolated values.

magnitude in both streamwise and normal directions. Large flow fluctuations are found only in pores that are directly exposed to the boundary layer, and the intensity decreases rapidly towards the airfoil chord line. This is also in line with the observations of Naaktgeboren et al. [39] and Dukhan and Minjeur [33] regarding the entrance length of a porous material that is approximately equal to one pore diameter away from the surface. Similar to the mean velocity trends, velocity fluctuations also tend to increase towards the TE tip. Lastly, the pressure fluctuations contour in shown in plot $(e)$; the values are normalized with freestream dynamic pressure $\left(q_{\infty}=0.5 \rho_{\infty} U_{\infty}^{2}\right)$. Pressure fluctuations are also relatively high within one unit-cell distance from the surface, and it is evident that the they are enhanced near the trailing edge. Overall, both velocity and pressure fluctuations in the boundary layer on the DMND TE have higher intensity compared to the SLD one, which is also previously indicated in Fig. 8

The application of the porous trailing edge has been reported to adversely affect the aerodynamic performance of the airfoil [40, 41]. To verify this, the mean drag coefficient of the diamond TE is compared with that of the solid TE in Table 4. The drag coefficient of an airfoil section has been estimated by measuring the velocity deficit in the airfoil wake as shown in equation 10 , following Faleiros et al. [42]. Velocity distribution is sampled at $x / c=4$, along $-2.5<y / c<2.5$, where at each location, the velocity time history is averaged over a period of 10 flow passes.

$$
C_{d}=2 \int_{-\infty}^{\infty}\left(1-\frac{U(y)}{U_{\infty}}\right)\left(\frac{U(y)}{U_{\infty}}\right) d y
$$

As shown in Table 4 , the airfoil with DMND TE produces slightly higher drag compared to the SLD one. This behavior can be associated with the higher shear stress near the pore entrance due to the flow transpiration through the porous medium [37] in addition to the increased surface roughness [40]. Moreover, based on the mean surface pressure distribution plot in Fig. 10 (a), it is arguable that the drag increase contribution mainly comes from the DMND TE, since the pressure distribution upstream of the solid-porous junction remains unchanged in comparison to the SLD TE. However, the figure also evidences that there is a slight increase of adverse pressure gradient upstream of the solid-porous junction, which is also responsible for enhancing the boundary layer growth on the DMND TE (see Fig. 7). Further downstream, however, the pressure gradient of both TE types is very similar.

As reported in literature [1, 11, 43, 44], the changes in the turbulence due to the presence of the porous trailing edge can be related to the noise radiation. For instance, TE noise intensity is known to be proportional to the intensity and spanwise coherence of surface pressure fluctuations following Amiet's model [1]. Fig. 10 (b) shows that the DMND TE 

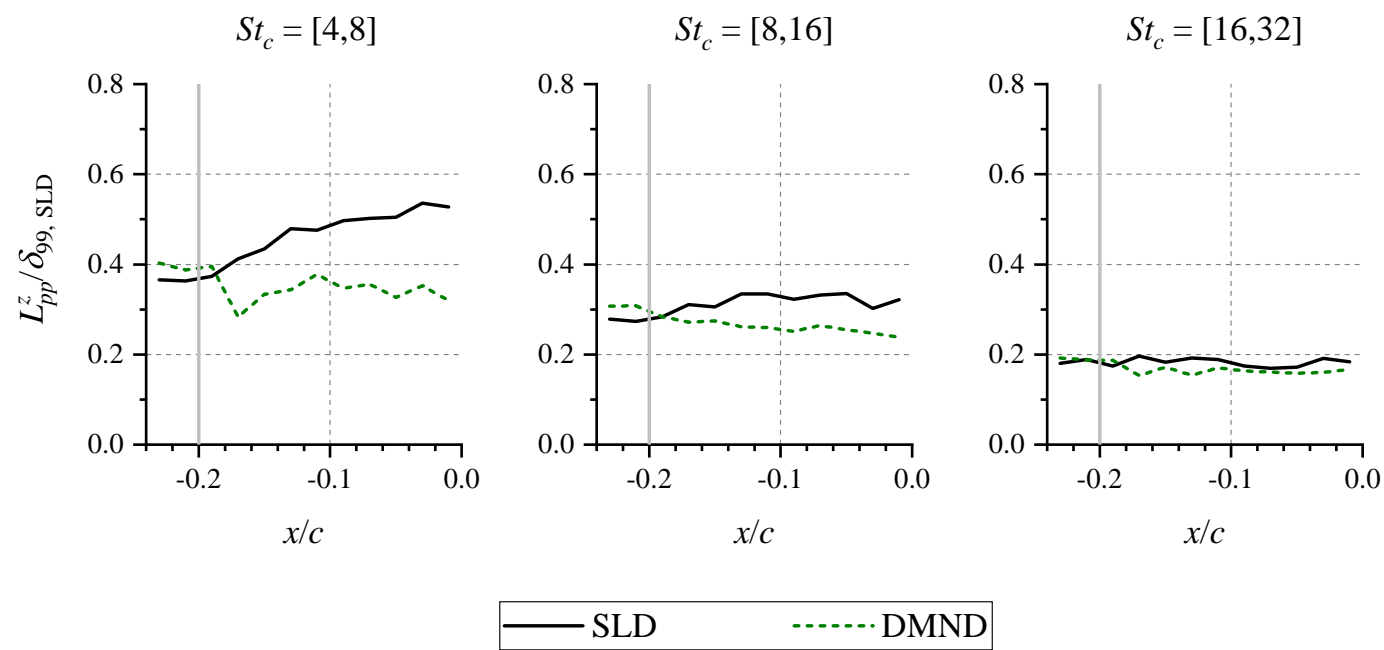

Fig. 11 Comparison of streamwise distribution of spanwise correlation length of surface pressure fluctuations $L_{p p}^{z}$ between the solid and porous TE case.

(a) SLD

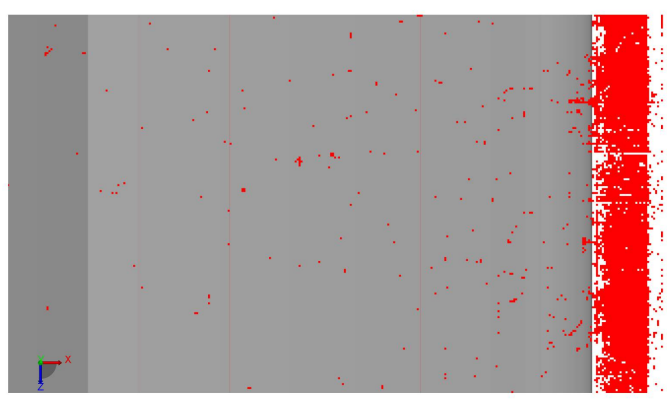

$S t_{c}=[8,16]$

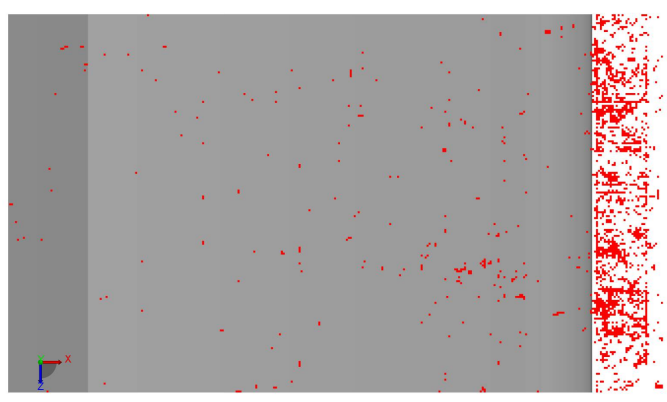

$S t_{c}=[16,32]$

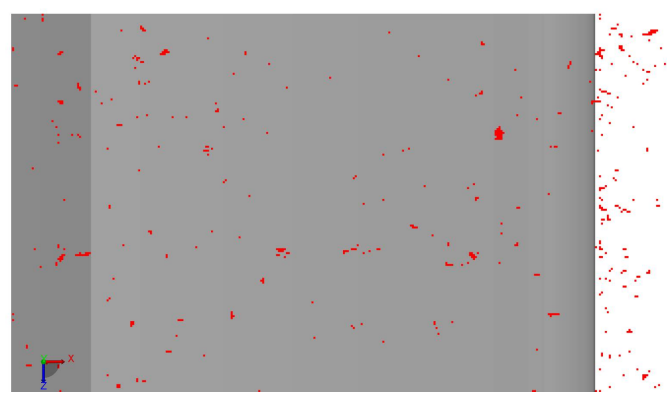

(b) DMND
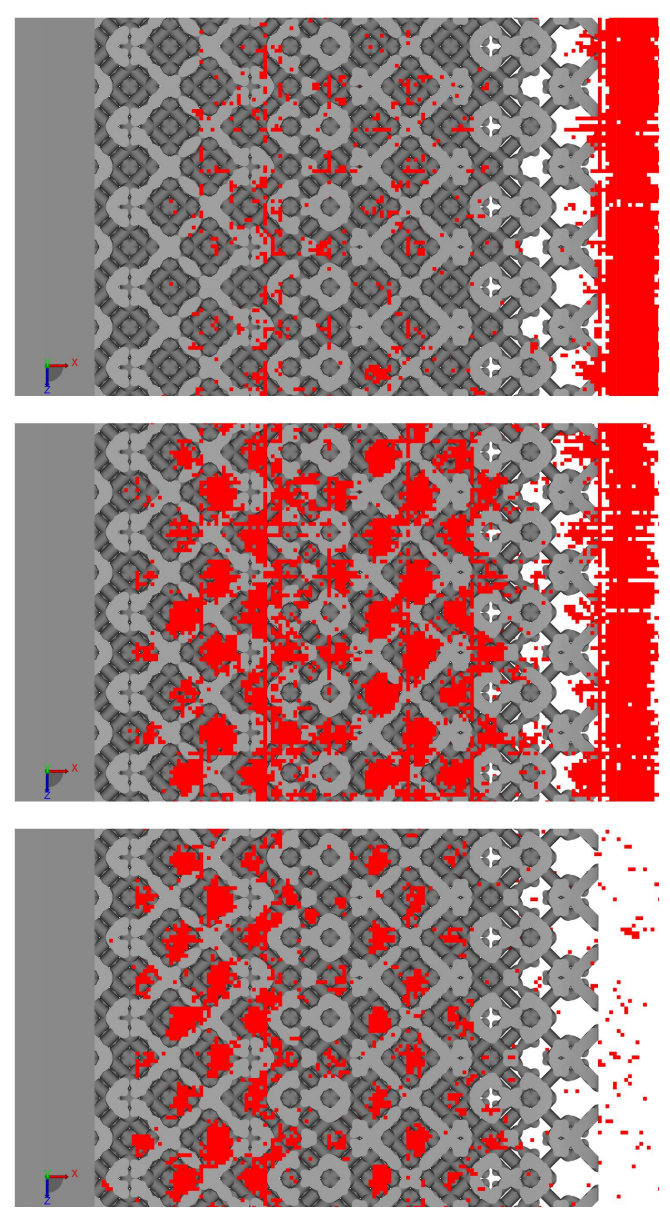

Fig. 12 Noise source localisation based on the vortex sound theory alogrithm, FIND. Fluid clusters corresponding to possible noise sources are shown in red. The last $20 \%$ of the airfoil chord is given light grey color. 
causes a substantial increase of the surface pressure fluctuations $C_{p}$, RMS in comparison to the SLD TE; at $x / c=0$, the $C_{p, \text { RMS }}$ of the DMND TE is $42 \%$ higher than the SLD one. The spanwise correlation length $L_{z, p p}$ along the TE region of the airfoil is plotted in Fig. 11. It is computed through the integration of the correlation decay in the spanwise direction with the midspan as the reference; the complete procedure is outlined in ref. [13]. The correlation length has been averaged across three different frequency ranges corresponding to the noise spectra previously shown in Fig. 5 (b). The correlation length upstream of the solid-porous junction is similar for both types of TE throughout the frequency range of interest. However, at $4<S t_{c}<16$, the $L_{z, p p}$ of the SLD TE increases gradually towards $x / c=0$ whereas that of the DMND TE slightly decreases. On average, the correlation length of the DMND TE is $35 \%$ lower at $S t_{c}=[4,8]$ and $17 \%$ at $S t_{c}=[8,16]$ with respect to the SLD one. Nevertheless, the $L_{z, p p}$ plot at the highest frequency band shows almost no difference. The reduction of spanwise correlation length caused by a permeable TE has also been reported in experiments [12, 44].

Despite having lower spanwise correlation length, the DMND TE increases the surface pressure fluctuations by a more significant proportion. Hence. following Amiet's model, the DMND TE is expected to produce more intense acoustic scattering than its solid counterpart, which contradicts the observed acoustic trends. In fact, as previously reported by the authors [13], the porous TE modifies the scattering characteristics of the airfoil, which eventually leads to noise attenuation. Additionally, it is possible to argue that the surface detail of the DMND TE is responsible for the high frequency noise increase. To verify this, a noise source localization method based on Howe's [45] vortex sound theory has been employed. This method is part of the PowerFLOW software and is referred to as FIND (Flow-Induced Noise Detection). It has also been previously employed for TE serrations study [46].

The FIND tool locates fluid regions with large vorticity gradient in the flow field. Such regions are shown in 12 for different frequency ranges. For the SLD TE, the noise sources are expected to be located near to the TE location, as shown in the figure. Their intensity becomes lower at higher frequencies, consistent with the noise spectra previously presented in Fig. 5. For the DMND TE, the trend appears to be quite different. At the lowest frequency band where noise reduction is observed, the figure suggests that the actual TE is where noise sources are mainly found; the solid-porous junction does not appear to produce noticeable noise sources. Considering that the shape of the TE of the DMND material is highly inhomogeneous with relatively small solid area, in addition to the pressure release process [16], the scattering efficiency at this location is likely to be lower compared to that of the SLD TE. Nevertheless, at higher frequencies where the excess noise starts appearing, noise sources can be found clustered in the open pores at the surface of the porous material. This can be related to Fig. $9(e)$ where regions of large pressure fluctuations are formed locally on the exposed pores. This observation supports the hypothesis that the high frequency noise increase is related to the geometrical detail at the surface of the porous medium, i.e., surface roughness noise [9, 10].

\section{Conclusion}

This manuscript has presented a numerical study on the application of a 3D-printed porous insert to reduce broadband trailing-edge noise. The porous material is based on a synthetic unit cell that resembles the lattice of diamond atoms, and the resulting porous insert is referred to as the diamond TE. The porous material is characterized using a pressure-drop test rig, which has also been replicated numerically. The geometry of the diamond TE has been fully resolved in the simulation. This porous insert is applied at the last $20 \%$ of the chord of a NACA 0018 airfoil. Simulation results are found to be in good agreement with the experiment. The diamond TE is found to reduce noise by up to $10 \mathrm{~dB}$ at low frequencies, but the noise attenuation becomes smaller above $S t_{c}=8$, and later followed by a slight noise increase in the frequency range above $S t_{c}=16$.

Flow field measurements show that the diamond insert causes the boundary layer to become thicker than that on the solid TE. Moreover, the intensity of flow fluctuations in the boundary layer are increased due to the material permeability. The contours of velocity statistics inside the porous trailing edge reveal that large flow fluctuations are limited to a distance of roughly 1 unit cell away from the surface. Beneath this "entrance length", the fluctuations intensity diminishes rapidly. The diamond trailing edge also causes a slight drag increase. The analysis of surface pressure statistics reveals that the diamond TE produces substantially higher surface pressure fluctuations and smaller spanwise correlation length with respect to those for the solid TE. Following noise prediction models for solid TE, the diamond TE would have generated higher noise intensity compared to the solid TE, which contradicts the acoustic results. Using the FIND algorithm, it is found that the higher surface pressure fluctuations of the diamond TE are responsible for the noise increase at high frequencies. This supports various past experimental observations which linked surface roughness noise to high-frequency noise increase. Hence, these findings corroborate the argument that the noise attenuation of the diamond TE is likely to be the result of differences in noise scattering characteristics between 
porous and solid TE.

The diamond TE has been found to produce similar aerodynamic and acoustic effects as the metal-foam-based porous TE which was previously studied by the authors, implying that material permeability is indeed the main driver behind these phenomena. Considering that the acoustic response from the usage of metal-foam trailing edge, which has random pore distribution, could be mimicked by a 3D-printed one with a regular unit cell, this study hints at the possibility of optimizing the unit cell design to achieve higher noise reduction at a wider range of frequencies, and to mitigate the aerodynamic penalty.

\section{Acknowledgments}

The authors would like to acknowledge the technical support of Dr. Wouter van der Welden from Dassault Systemes regarding the PowerFLOW template for the airfoil trailing-edge noise study.

We acknowledge that the results of this research have been achieved using the Distributed European Computing Initiative (DECI-15) resources Salomon at the National Supercomputing Center (IT4Innovations) based in Ostrava, Czech Republic with the support from the Partnership for Advanced Computing in Europe (PRACE).

This study is also supported by the project SMARTANSWER (Smart Mitigation of flow-induced Acoustic Radiation and Transmission for reduced Aircraft, surface traNSport, Workplaces and wind enERgy noise) which has received funding from the European Union's Horizon 2020 research and innovation program under the Marie Skłodowska-Curie grant agreement No. 722401. More information can be found on https://www.h2020-smartanswer.eu/.

\section{References}

-[1] Amiet, R. K., "Noise due to turbulent flow past a trailing edge," Journal of sound and vibration, Vol. 47, No. 3, 1976, pp. 387-393.

- [2] Howe, M. S., "A review of the theory of trailing edge noise," Journal of sound and vibration, Vol. 61, No. 3, 1978, pp. 437-465.

-[3] Oerlemans, S., Fisher, M., Maeder, T., and Kögler, K., "Reduction of wind turbine noise using optimized airfoils and trailing-edge serrations," AIAA journal, Vol. 47, No. 6, 2009, pp. 1470-1481.

[4] Chase, D. M., "Noise radiated from an edge in turbulent flow," AIAA journal, Vol. 13, No. 8, 1975, pp. $1041-1047$.

[5] Gruber, M., Joseph, P., and Chong, T. P., "Experimental investigation of airfoil self noise and turbulent wake reduction by the use of trailing edge serrations," 16th AIAA/CEAS aeroacoustics conference, 2010, p. 3803.

-[6] Chong, T. P., Vathylakis, A., Joseph, P. F., and Gruber, M., "Self-noise produced by an airfoil with nonflat plate trailing-edge serrations," AIAA journal, Vol. 51, No. 11, 2013, pp. 2665-2677.

-[7] Herr, M., and Dobrzynski, W., "Experimental Investigations in Low-Noise Trailing Edge Design.” AIAA journal, Vol. 43, No. 6, 2005, pp. 1167-1175.

- [8] Geyer, T., Sarradj, E., and Fritzsche, C., "Measurement of the noise generation at the trailing edge of porous airfoils," Experiments in Fluids, Vol. 48, No. 2, 2010, pp. 291-308.

-[9] Geyer, T. F., and Sarradj, E., "Trailing edge noise of partially porous airfoils," 20th AIAA/CEAS Aeroacoustics Conference, 2014, p. 3039.

[10] Rubio Carpio, A., Merino Martinez, R., Avallone, F., Ragni, D., Snellen, M., and van der Zwaag, S., "Broadband Trailing-Edge Noise Reduction Using Permeable Metal Foams," INTER-NOISE and NOISE-CON Congress and Conference Proceedings, Vol. 255, Institute of Noise Control Engineering, 2017, pp. 2755-2765.

-[11] Rubio Carpio, A., Martínez, R. M., Avallone, F., Ragni, D., Snellen, M., and van der Zwaag, S., "Experimental characterization of the turbulent boundary layer over a porous trailing edge for noise abatement," Journal of Sound and Vibration, Vol. 443, 2019, pp. 537-558.

[12] Rubio Carpio, A., Avallone, F., Ragni, D., Snellen, M., and van der Zwaag, S., "Mechanisms of broadband noise generation on metal foam edges," Physics of Fluids, Vol. 31, No. 10, 2019, p. 105110.

[13] Teruna, C., Manegar, F., Avallone, F., Ragni, D., Casalino, D., and Carolus, T., "Noise reduction mechanisms of an open-cell metal-foam trailing edge," Journal of Fluid Mechanics, Vol. 898, 2020. 
[14] Rubio Carpio, A., Avallone, F., and Ragni, D., "On the Role of the Flow Permeability of Metal Foams on Trailing Edge Noise Reduction,” 2018 AIAA/CEAS Aeroacoustics Conference, 2018, p. 2964.

-[15] Sarradj, E., and Geyer, T., "Noise generation by porous airfoils," 13th AIAA/CEAS Aeroacoustics Conference (28th AIAA Aeroacoustics Conference), 2007, p. 3719.

[16] Delfs, J., Faßmann, B., Lippitz, N., Lummer, M., Mößner, M., Müller, L., Rurkowska, K., and Uphoff, S., “SFB 880: Aeroacoustic research for low noise take-off and landing," CEAS Aeronautical Journal, Vol. 5, No. 4, 2014 , pp. $403-417$.

[17] Ali, S. A. S., Azarpeyvand, M., and da Silva, C. R. I., "Trailing-edge flow and noise control using porous treatments," Journal of Fluid Mechanics, Vol. 850, 2018, pp. 83-119.

-[18] Mößner, M., and Radespiel, R., "Modelling of turbulent flow over porous media using a volume averaging approach and a Reynolds stress model," Computers \& Fluids, Vol. 108, 2015, pp. 25-42.

-[19] Teruna, C., Manegar, F. A., Avallone, F., Casalino, D., Ragni, D., Rubio Carpio, A., and Carolus, T., "Numerical Analysis of Metal-Foam Application for Trailing Edge Noise Reduction,” 25th AIAA/CEAS Aeroacoustics Conference, 2019, p. 2650.

[20] Succi, S., The Lattice Boltzmann Equation: for Fluid Dynamics and Beyond, Oxford university press, 2001.

[21] Avallone, F., van der Velden, W., Ragni, D., and Casalino, D., "Noise reduction mechanisms of sawtooth and combed-sawtooth trailing-edge serrations," Journal of Fluid Mechanics, Vol. 848, 2018, pp. 560-591.

[22] Teruna, C., Casalino, D., Ragni, D., and Avallone, F., "Numerical Analysis of a Linear Cascade Model for Rotor-Stator Interaction Aeroacoustics," 2018 AIAA/CEAS Aeroacoustics Conference, 2018, p. 4189.

-[23] Chen, H., Chen, S., and Matthaeus, W. H., "Recovery of the Navier-Stokes equations using a lattice-gas Boltzmann method," Physical Review A, Vol. 45, No. 8, 1992, p. R5339.

-[24] Bhatnagar, P. L., Gross, E. P., and Krook, M., "A model for collision processes in gases. I. Small amplitude processes in charged and neutral one-component systems," Physical review, Vol. 94, No. 3, 1954, p. 511.

[25] Yakhot, V., and Orszag, S. A., "Renormalization group analysis of turbulence. I. Basic theory," Journal of scientific computing, Vol. 1, No. 1, 1986, pp. 3-51.

[26] Launder, B. E., and Spalding, D. B., "The numerical computation of turbulent flows," Numerical Prediction of Flow, Heat Transfer, Turbulence and Combustion, Elsevier, 1983, pp. 96-116.

-[27] Williams, J. F., and Hawkings, D. L., "Sound generation by turbulence and surfaces in arbitrary motion," Phil. Trans. R. Soc. Lond. A, Vol. 264, No. 1151, 1969, pp. 321-342.

[28] Farassat, F., and Succi, G. P., "A review of propeller discrete frequency noise prediction technology with emphasis on two current methods for time domain calculations," Journal of Sound and Vibration, Vol. 71, No. 3, 1980, pp. $399-419$.

[29] Casalino, D., "An advanced time approach for acoustic analogy predictions," Journal of Sound and Vibration, Vol. 261, No. 4, 2003, pp. 583-612.

[30] Ingham, D. B., and Pop, I., Transport phenomena in porous media, Elsevier, 1998.

-[31] Liu, H., Azarpeyvand, M., Wei, J., and Qu, Z., “Tandem cylinder aerodynamic sound control using porous coating,” Journal of Sound and Vibration, Vol. 334, 2015, pp. 190-201.

[32] Rubio Carpio, A., Avallone, F., Ragni, D., Snellen, M., and van der Zwaag, S., "3D-printed perforated trailing edges for broadband noise abatement," 25th AIAA/CEAS Aeroacoustics Conference, 2019, p. 2458.

-[33] Dukhan, N., and Minjeur, C., "Minimum Thickness for Open-Cell Metal Foam to Behave as a Porous Medium," 40th Fluid Dynamics Conference and Exhibit, 2010, p. 4618.

[34] Baril, E., Mostafid, A., Lefebvre, L.-P., and Medraj, M., "Experimental Demonstration of Entrance/Exit Effects on the Permeability Measurements of Porous Materials,” Advanced Engineering Materials, Vol. 10, No. 9, 2008 , p. 889-894. https://doi.org/10.1002/adem.200800142

[35] Welch, P., "The use of fast Fourier transform for the estimation of power spectra: a method based on time averaging over short, modified periodograms," IEEE Transactions on audio and electroacoustics, Vol. 15, No. 2, 1967, pp. 70-73. 
[36] León, C. A., Merino-Martínez, R., Ragni, D., Avallone, F., and Snellen, M., "Boundary layer characterization and acoustic measurements of flow-aligned trailing edge serrations," Experiments in Fluids, Vol. 57, No. 12, 2016, p. 182.

[37] Jimenez, J., Uhlmann, M., Pinelli, A., and Kawahara, G., "Turbulent shear flow over active and passive porous surfaces," Journal of Fluid Mechanics, Vol. 442, 2001, pp. 89-117.

[38] Flack, K. A., and Schultz, M. P., "Roughness effects on wall-bounded turbulent flows," Physics of Fluids, Vol. 26, No. 10, 2014, p. 101305.

[39] Naaktgeboren, C., Krueger, P., and Lage, J., "Limitations of Darcy’s Law in Light of Inlet and Exit Pressure Drops," International Conference on Applications of Porous Media, 2004.

-[40] Geyer, T., Sarradj, E., and Fritzsche, C., "Porous airfoils: noise reduction and boundary layer effects," International journal of aeroacoustics, Vol. 9, No. 6, 2010, pp. 787-820.

-[41] Herr, M., Rossignol, K.-S., Delfs, J., Lippitz, N., and Mößner, M., "Specification of Porous Materials for Low-Noise Trailing-Edge Applications," 20th AIAA/CEAS Aeroacoustics Conference, American Institute of Aeronautics and Astronautics, 2014. https://doi.org/10.2514/6.2014-3041, URL http://arc.aiaa.org/doi/10.2514/6.2014-3041

- [42] Faleiros, D. E., Tuinstra, M., and Hoeijmakers, H., "Drag, lift and effective angle of attack from analysis wake of an airfoil in open-jet wind tunnel," 54th AIAA Aerospace Sciences Meeting 2016: 4-8 January 2016, San Diego, California, USA, American Institute of Aeronautics and Astronautics Inc.(AIAA), 2016.

[43] Brooks, T. F., Pope, D. S., and Marcolini, M. A., "Airfoil Self-Noise and Prediction,” NASA Reference Publication, NASA-RP1218, 1989.

[44] Ananthan, V., Bernicke, P., Akkermans, R., Hu, T., and Liu, P., "Effect of porous material on trailing edge sound sources of a lifting airfoil by zonal Overset-LES," Journal of Sound and Vibration, 2020, p. 115386.

[45] Howe, M. S., Theory of vortex sound, Vol. 33, Cambridge university press, 2003.

[46] Avallone, F., Van der Velden, W., and Ragni, D., "Benefits of curved serrations on broadband trailing-edge noise reduction," Journal of Sound and Vibration, Vol. 400, 2017, pp. 167-177. 